\title{
Laparoscopic Surgical Skills programme: preliminary evaluation of Grade I Level 1 courses by trainees
}

\author{
Sonja Buzink ${ }^{1,2}$, Marek Soltes ${ }^{3}$, Jozef Radonak ${ }^{3}$, Abe Fingerhut ${ }^{4}$, George Hanna ${ }^{5}$, Jack Jakimowicz ${ }^{1,2}$ \\ ${ }^{1}$ Faculty of Industrial Design Engineering, Delft University of Technology, Delft, the Netherlands \\ ${ }^{2}$ Catharina Hospital Eindhoven, Eindhoven, the Netherlands \\ 31st Department of Surgery, University of Pavol Jozef Safarik, Kosice, Slovak Republic \\ ${ }_{4}^{4} 1^{\text {st }}$ Department of Surgery, Hippocration Hospital, University of Athens Medical School, Athens, Greece \\ ${ }^{5}$ Department of Surgery and Cancer, St. Mary's Hospital, Imperial College, London, United Kingdom
}

Videosurgery Miniinv 2012; 7 (3): 188-192 DOI: 10.5114/wiitm.2011.28895

\begin{abstract}
Introduction: New training models are needed to maintain safety and quality of surgical performance. A simulated setting using virtual reality, synthetic, and/or organic models should precede traditional supervised training in the operating room. Aim: The aim of the paper is to describe the Laparoscopic Surgical Skills (LSS) programme and to provide information about preliminary evaluation of Grade I Level 1 courses, including overall quality, applicability of the course content in practice and the balance between theory and hands-on training modules, by participating trainees.

Material and methods: During 5 accredited LSS Grade I Level 1 courses held in Eindhoven (the Netherlands), Kosice (Slovak Republic), and Lisbon (Portugal) between April 2011 and January 2012, demographic data and pre-course surgical experience in laparoscopic surgery of the participants were recorded. The final course evaluation form was completed by each participant after the course (anonymous) to evaluate course progress, course materials, assessment, staff, location and overall impression of the course on a 1-10 scale to obtain feedback information.

Results: Forty-seven surgeons of 5 different nationalities were enrolled in an LSS Grade I Level 1 programme. Most participants were first or second year residents $(n=25)$, but also already established surgeons took part $(n=6)$. The mean age of the participants was 31.2 years $(S D=2.86)$, the male/female ratio was $32 / 15$, and previous experience with laparoscopic surgery was limited. Overall impression of the course was rated with 8.7 points $(S D=0.78)$. The applicability of the course content in practice and the balance between theory and hands-on training were also rated very well - mean $8.8(S D=1.01)$ and 8.1 points $(S D=0.80)$ respectively.

Conclusions: Laparoscopic Surgical Skills Grade I Level 1 courses are evaluated as well balanced, with content applicable in clinical practice, meeting the expectations of individual participants. International interest in the programme suggests that LSS might become the future European standard in surgical education in laparoscopic surgery. Further conclusions concerning success of the programme may be drawn after the completion of clinical assessment of enrolled participants.
\end{abstract}

Key words: laparoscopy, training, simulation, virtual reality.

\section{Introduction}

Considering safety and quality of surgical performance, there is an urgent need to improve train- ing, assessment and accreditation for technologydependent surgical procedures. Established traditional, residency-based master-apprenticeship models appear unsuitable for training in laparoscopy, espe- 
cially in early stages of surgical education. New working time restrictions in combination with medicolegal and socio-economic considerations have considerably reduced the amount of time available to train and supervise trainees [1]. Furthermore, the concept of accreditation based only on the number of procedures performed does not seem to be acceptable any longer. All of this together has led to a paradigm shift in surgical training [2]. Currently, a simulated setting using virtual reality, synthetic, and/or organic models should be the first stage of education in laparoscopic surgery, thus bringing the training process out of the operating room. The crucial clinical training stage, during which trainees operate on patients under the supervision of an experienced surgeon, should only follow as the second step after successful completion of simulation training. The Laparoscopic Surgical Skills (LSS) programme, developed under the auspices of the European Association for Endoscopic Surgery (EAES), aims to provide a standard to credential surgeons to perform laparoscopic surgery effectively and safely, meeting the requirements of both surgeons and health care authorities [3].

\section{Aim}

The aim of the paper is to describe the overall outline of the LSS programme and to provide information about preliminary evaluation of Grade I Level 1 courses, concerning overall quality, applicability of the course content in practice, and the balance between theory and hands-on training modules, by participating trainees.

\section{Material and methods}

\section{Outline of Laparoscopic Surgical Skills}

Laparoscopic Surgical Skills offers a standard for comprehensive performance assessment for training and education in laparoscopic surgery within a multilevel curriculum combining criterion-based assessment in the skills lab with clinical assessment of performance on indicator procedures. Within each LSS level, the assessment incorporates a sequence of tests to evaluate a surgeon's proficiency in cognitive skills, surgical technical skills, and judgment. Laparoscopic Surgical Skills is developed for surgeons in training, surgical fellows, practising surgeons and other physicians who perform laparoscopic surgery or would like to do so. Eligible candidates can enrol either for the LSS assessment or for an LSS accredited course, of which the LSS assessment is an integral part. This provides surgeons of all levels of expertise the opportunity to efficiently obtain LSS certification.

To certify that the participating surgeons have reached the appropriate level of performance to obtain the LSS diploma, they need to pass several types of performance assessments (Figure 1). All LSS assessments are criterion-based and very practice-oriented.

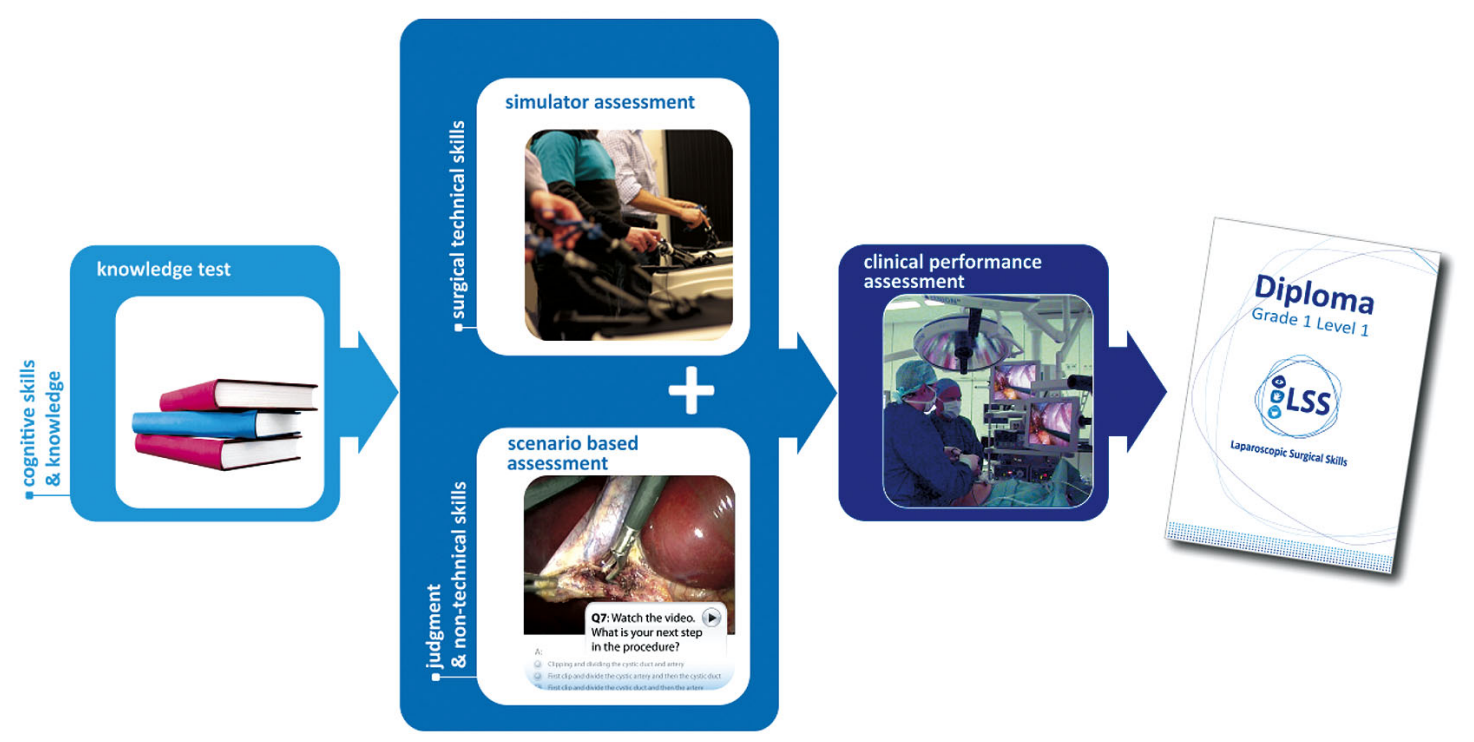

Figure 1. Schematic overview of LSS assessment process 


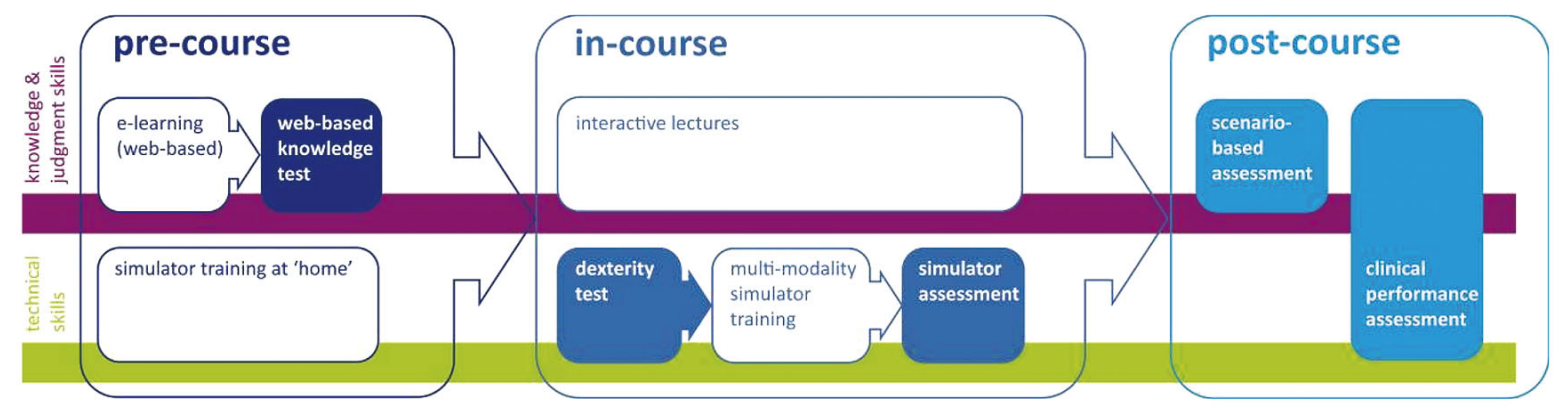

Figure 2. Structure of LSS accredited courses

With a pre-course knowledge test, adequate acquaintance of the theory presented in the course documents (pre-course learning) regarding procedures, techniques, instrumentation and ergonomics is tested. Passing the web-based exam is a requirement to be admitted to the scenario-based assessment and simulator assessment.

Simulator assessment certifies that participants have achieved a sufficient level of psychomotor and technical surgical skills to start performing the specific index procedures in the clinical setting under the supervision of an acknowledged trainer. To pass the simulator assessment successfully, the participant needs to demonstrate sufficient performance of a selection of basic and procedural tasks on the assessment simulator. Passing the simulator assessment is required for admission to the clinical performance assessment.

Scenario-based assessment is conducted to assess the knowledge and judgment skills with regard to the index procedures. The foundation of the assessment is based on the theory, procedural training and expert discussions as offered in LSS accredited courses. In the test several real life scenarios are presented with problems or questions at specific critical moments, which the participant has to solve successfully. Passing the scenario-based assessment is required for admission to the clinical performance assessment.

After successful completion of the simulator assessment and scenario-based assessment, evidence for sufficient expertise in supervised clinical procedures is required. Each LSS level is therefore concluded by two steps for clinical performance assessment. The Global Assessment Score (GAS) forms [4] are used to assess overall workplace performance in the index procedures, after which the
Competency Assessment Tool (CAT) [5] is used to analyse video recordings of two full-length recorded index procedures.

Established surgeons holding a national board certification of completion of training (General Surgery), or an equivalent national diploma that enables them to practice independently, are required to submit logbook evidence of laparoscopic procedures performed. The core LSS faculty will then decide on an individual basis if they should enrol for the full assessment process or are directly eligible for the video assessment.

\section{Laparoscopic Surgical Skills Grade I Level 1}

Laparoscopic Surgical Skills accredited courses follow a goal-oriented and criterion-based approach that takes the training needs of each individual participant into account. They are constructed around a vast amount of training on a combination of simulation tools and interactive expert discussions, offering a well-balanced mix of hands-on training and application of theory (Figure 2). Virtual reality simulation tools, box trainers (with synthetic or organic tissue) and augmented reality simulation tools are utilized in the courses. This variation enlarges the transferability of skills and keeps repetitive training of basic skills interesting [6]. The hands-on training in the course is criterion-based to enlarge the effectiveness and efficiency of the training and the engagement of the trainees in their training $[7,8]$. The LSS assessment is an integral part of the course. There are specific accreditation requirements that need to be fulfilled to become an LSS accredited training centre to warrant the contents and quality of LSS accredited courses and the LSS assessment. Laparoscopic Surgical Skills Grade I Level 1 is focused on diagnostic 
laparoscopy, laparoscopic cholecystectomy and laparoscopic appendicectomy.

\section{Course evaluation}

Demographic data collection was performed prospectively: data on age, sex, nationality, year of surgical training and pre-course surgical experience in laparoscopic surgery were recorded prior to the course. The final course evaluation form was completed by each participant after the course (anonymously) to evaluate course progress, course materials, assessment, staff, location and overall impression of the course on a scale of 1-10 (1- abominable, 2 - very poor, 3 - poor, 4 - very unsatisfactory, 5 - unsatisfactory, 6 - satisfactory, 7 - more than satisfactory, 8 - good, 9 - very good, 10 - excellent).

\section{Results}

So far, 5 accredited LSS Grade I Level 1 courses have been offered in Eindhoven (the Netherlands), Kosice (Slovak Republic), and Lisbon (Portugal) between April 2011 and January 2012. Forty-seven surgeons of 5 different nationalities took part in one of the above-mentioned courses and were enrolled in an LSS Grade I Level 1 programme. Most participants were first or second year residents $(n=25)$, but also already established surgeons took part $(n=6)$. The clinical experience in laparoscopy was rather limited (Figure 3) and in general matched the expertise level for which LSS Grade I Level 1 has been developed. The mean age of the participants was 31.2 years $(S D=2.86)$ and the male/female ratio was $32 / 15$. All 47 participants completed the knowledge test, scenario-based assessment, and simulator assessment and are currently in the phase of clinical training and assessment.

Feedback of the participants of LSS Grade I Level 1 accredited courses was generally positive. Overall impression of the course was rated 8.7 points $(S D=0.78)$ on a scale of 10 . The applicability of the course content in practice and the balance between theory and hands-on training were also rated very well - mean $8.8(S D=1.01)$ and 8.1 points $(S D=0.80)$ respectively.

\section{Discussion}

While the higher levels of the LSS programme are still in the development phase, the LSS Grade I Level 1

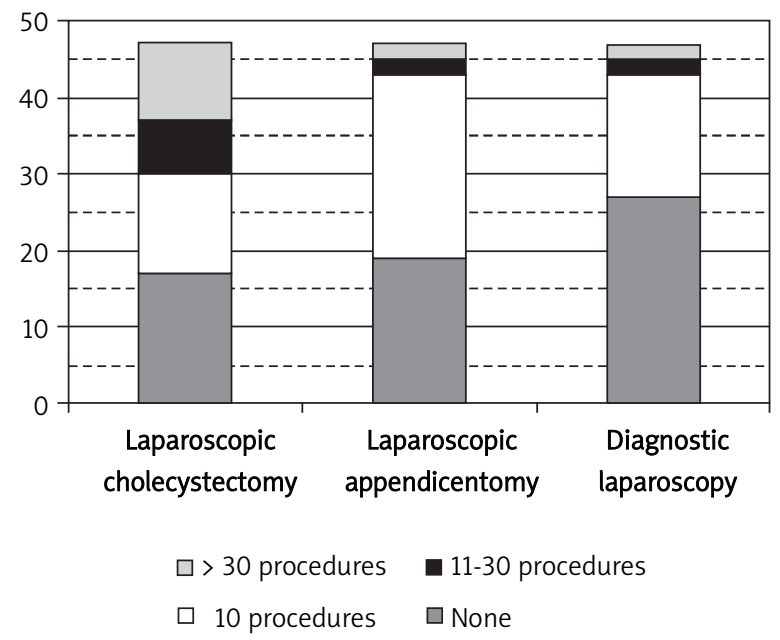

Figure 3. Number of laparoscopic procedures performed prior to the course

is running in more and more training centres across Europe. It appears that the structure of the training, applicability of the course content in practice and the proportion of theory and hands-on training are rated positively by the trainees. The LSS programme appeals to both residents in training and already accredited surgeons. International participation indicates that the LSS programme has the potential to become a future European standard in surgical education in laparoscopic surgery. Further conclusions concerning success of the programme may be drawn after the completion of clinical assessment of enrolled participants.

\section{Acknowledgments}

We thank Simbionix Ltd. for providing LapMentor systems and technical support during the accredited courses. Our gratitude goes to Karl Storz GmbH \& Co. KG for providing a generous educational grant to the LSS programme and equipment support for the accredited courses. The LSS accredited courses were additionally supported by Covidien (Eindhoven) and Ethicon (Kosice and Lisbon). A research grant was provided by the European Association for Endoscopic Surgery (EAES) for the development of LSS. The work of S. N. Buzink is funded by a Fellowship grant provided by Delft University of Technology. The work of $\mathrm{M}$. Soltes and J. Radonak is funded by a KEGA Grant 017UPJS-4/2011. 


\section{References}

1. Jakimowicz J, Fingerhut A. Simulation in surgery. Br J Surg 2009; 96: 563-4.

2. Stefanidis D. Optimal acquisition and assessment of proficiency on simulators in surgery. Surg Clin North Am 2010; 90: 475-89.

3. Laparoscopic Surgical Skills Foundation 2011; available from: www.lss-surgical.eu

4. Wyles S, Miskovic D, Ni Z, et al. Analysis of laboratory-based laparoscopic colorectal surgery workshops within the English National Training Programme. Surg Endosc 2011; 25: 1559-66.

5. Miskovic D. Laparoscopic Colorectal Competency Assessment Tool (LCAT) for the National Training Program. Annual Meeting of the American Society of Colon and Rectal Surgeons 2010: Minneapolis, MN.

6. Brinkman WM, Havermans SY, Buzink SN, et al. Single versus multi modality training basic laparoscopic skills. Surg Endosc 2012; 26: 2172-8.

7. Brinkman WM, Buzink SN, Alevizos, et al. Criterion-based laparoscopic training reduces total training time. Surg Endosc 2011; 25: 3526-30.

8. Stefanidis D, Acker CE, Greene FL. Performance goals on simulators boost resident motivation and skills laboratory attendance. J Surg Educ 2010; 67: 66-70. 